\title{
Al in Germany: Well-Prepared and Eager to Do Something
}

\section{Daniel Sonntag ${ }^{1}$}

๑) Springer-Verlag GmbH Germany, part of Springer Nature 2018
Dear readers,

Ready, willing and able? That's how the British select committee on artificial intelligence, by the Authority of the House of Lords, sets the title of their report which was published in April 2018. It says: "Our inquiry has concluded that the UK is in a strong position to

be among the world leaders in the development of artificial intelligence during the twenty-first century. Britain contains leading AI companies, a dynamic academic research culture, a vigorous start-up ecosystem and a constellation of legal, ethical, financial and linguistic strengths located in close proximity to each other. Artificial intelligence, handled carefully, could be a great opportunity for the British economy. In addition, AI presents a significant opportunity to solve complex problems and potentially improve productivity, which the UK is right to embrace". A well-prepared and eager to do something AI society in Germany should have the same characteristics and qualities.

Later in this report: "On the basis of the evidence we have received, we are convinced that vague statements about the UK 'leading' in AI are unrealistic and unhelpful, especially given the vast scale of investment in AI by both the USA and China. By contrast, countries such as Germany and Canada are developing cohesive strategies which take account of their circumstances and seek to play to their strengths as a nation". This sounds much clearer: for "Kuenstliche Intelligenz" in Germany, they refer for example to the German

Daniel Sonntag

Daniel.Sonntag@dfki.de

1 German Research Center for Artificial Intelligence (DFKI), Saarbrücken, Germany
Research Center for Artificial Intelligence, to offer valuable lessons as to how a national research centre should be operated and that Germany's approach is strongly influenced by its flagship Industrie 4.0 strategy for smart manufacturing (after an interview with Wolfgang Wahlster).

However, it should be allowed to ask, what do they and we think of the Brexit deal so far in this regard? I think that in order to find ways to circumvent Brexit and similar consequences based on (upcoming) political decisions, connecting AI research in Europe should be one of the cornerstones. Complementary to ECCAI, the representative body for the European artificial intelligence community, our German KI Journal has worked towards internationalisation over the last several years with its rigorous internationalisation strategy, to publish only English articles, to include international guest editors, and to appoint German editorial board members who live in other European countries and the US to act as our ambassadors for AI in Germany. This development has been initiated by the former editor-in-chief, Klaus Althoff, and Joachim Hertzberg who came up with the idea of what we now call AI on demand, to develop synergies among all the existing initiatives and to optimise Europe's potential, but rooted in the idea that AI technology should be publicly accessible and more accessible to the public. The KI Journal downloads have increased from 10 thousand to 40 thousand downloads since 2010. I hope we can continue this excellent development and give the German AI society a significant opportunity to publish new research, to connect and to develop a growing research and development perspective, turning academic research into commercial potential. In fact, my greatest hope is that the KI Journal can become the mouthpiece of the European AI community. Recent developments support this idea: our Chancellor Angela Merkel recently announced that the new federal government is planning an international AI centre with France.

Towards the goal of internationalisation in Europe, the editors are in the process of adapting and creating new submission categories, AI Transfer for example. It has been created in order to put more focus on current developments that 
have made their way out of research laboratories. We aim at descriptive articles accessible to the AI community at large, work that is strongly grounded in the needs of end users, or innovative applications in general.

Further developments of the KI Journal, together with the appointment of three new editorial board members, Kristian Kersting, Lars Kunze and Marco Ragni, have been fuelled by recent successes in machine learning, robotics and cognitive systems. The full list of new categories comprise of technical contributions (contact Bernd Ludwig, Lars Kunze or Kristian Kersting), system descriptions (contact Anni-Yasmin Turhan), project reports (contact Sabine Timpf or Christian Igel), dissertation and habilitation abstracts (contact Anni-Yasmin Turhan or Marco Ragni), AI Transfer (contact Daniel Sonntag), discussions (contact Sabine Timpf), interviews (contact Ubbo Visser), and news (contact Britta Wrede).

The European perspective, Artificial Intelligence for Europe, as published by the European Commission a few weeks ago, concludes that, like the steam engine or electricity in the past, $\mathrm{AI}$ is transforming our world, our society and our industry. In addition, the Call for the Establishment of a Confederation of Laboratories for Artificial Intelligence Research in Europe (CLAIRE), has been created recently. With your high-quality contributions to this new international KI Journal you contribute to a solid national and European AI framework. Thank you!

Daniel Sonntag

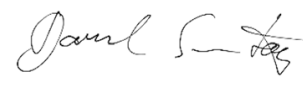

\section{Forthcoming Special Topics}

\subsection{Intentional Forgetting}

\section{Guest Editors \\ Christoph Beierle (FernUniversität in Hagen, Germany) \\ Ingo J. Timm (Trier University, Germany)}

Current trends, like the digital transformation and ubiquitous computing, yield in a massive increase in available data and information. On the other hand, in Artificial Intelligence systems, capacity of knowledge bases is limited due to computational complexity of many inference algorithms. Consequently, continuously sampling information and unfiltered storing in knowledge bases does not seem to be a promising or even feasible strategy.
In human evolution, learning and forgetting have evolved as advantageous strategies for coping with available information by adding new knowledge to and removing irrelevant information from the human memory. Learning has been adopted in AI systems in various algorithms and applications. Forgetting, however, especially intentional forgetting, has gained much less attention, despite the potential forgetting can provide for coping with the seemingly ever increasing information overload observed in current systems.

The aim of this special issue is to provide an overview on available theories and methods as well as ongoing research on forgetting and intentional forgetting relevant for the theory and practice of AI and AI systems from different perspectives like, e.g., knowledge representation, cognition, ontologies, reasoning, machine learning, self-organization, distributed AI, etc.

Topics

Submissions are solicited in all areas of forgetting and intentional forgetting relevant for AI, including but not restricted to:

- Foundations of (intentional) forgetting and terminology.

- Formal considerations/ Specification of (intentional) forgetting.

- Operationalization of (intentional) forgetting in AI systems.

- Complexity and efficiency considerations in reasoning.

- Cognitive aspects of (intentional) forgetting for AI systems.

- Applications with concepts of (intentional) forgetting.

We welcome submissions from any fields of AI, e.g.,

- Knowledge representation and belief revision.

- Knowledge representation and belief revision.

- Cognitive systems.

- Ontologies.

- Reasoning.

- Planning.

- Machine learning and data mining.

- Self-organization.

- Distributed AI and intelligent agents.

Submissions should be submitted either as:

- Technical contribution (6-10 pages).

- System description (4-6 pages).

- Project reports (4-6 pages).

- Discussion pieces. 
If you consider submitting a paper or if you have any questions regarding this special issue, please, contact the guest editors (beierle@fernuni-hagen.de).

\subsection{Smart Production}

\section{Guest Editors}

Martin Ruskowski (TU Kaiserslautern), Michael Beetz (Universität Bremen) and Georg Bartels (Universität Bremen)

Smart Production is key for industrial and societal growth, providing opportunities for designing our future work life. This special issue targets to illustrate the requirements and challenges of a smart production which targets not only cyber-physical systems as enabler technology and AIbased robots in production but also solutions for supporting humans in production. For a beneficial use of AI, the necessary infrastructure must be provided. This includes currently used communication protocols and proposed architectures, as well as production systems. Also, contributions concerning the role of AI in context of robots and human workers, respectively, and industrial use-cases are invited.

Contributions to the following topics are sought:

- Methodological and technological prerequisite for smart production from an AI perspective.

- Lean automation as a base concept for self-organizing factory.

- Edge computing and architecture.

- Intelligent production control/semantic OPC-UA.

- Base system for production plants for an efficient variability of a production process.

- Current AI applications from research and industry.

- Smart HMI.

- Advanced analytics und predictive maintenance.

- AI-based robotics in production.

- Robot assembly/disassembly planning.

- Knowledge representation and processing for robots.

- Cognition-enabled manipulation.

- Robot safety.

- Robotic logistics processes.

- Perception for grasping and manipulation.

- Force and tactile sensing in robot assembly.

- Compliant assembly. 